X. Palomer $\cdot$ L. Calpe-Berdiel $\cdot$ J. Verdaguer $\cdot$

J. Carrillo - X. Pastor - D. Mauricio - F. Blanco-Vaca

\title{
Atorvastatin does not decrease or delay diabetes onset in two different mouse models of type 1 diabetes
}

Received: 1 March 2005 / Accepted: 22 March 2005 / Published online: 30 June 2005

(C) Springer-Verlag 2005

Keywords Atorvastatin - Type 1 diabetes

Abbreviations HMG-CoA: 3-hydroxy-3-methylglutaryl coenzyme A - NOD: non-obese diabetic $\cdot$ Th: T helper

To the Editor:

Statins are 3-hydroxy-3-methylglutaryl coenzyme A (HMG-CoA) reductase inhibitors that are being extensively used for the treatment of hypercholesterolaemia in clinical practice. Competitive inhibition of HMG-CoA reductase by statins reduces, apart from cholesterol, the synthesis of isoprenoid intermediates, such as dolichol, ubiquinone, farnesol and geranylgeraniol, which are involved in posttranslational protein modification. This impairment of isoprenoid synthesis has been suggested to form the basis for the pleiotropic effects of statins, which include their anti-

X. Palomer · L. Calpe-Berdiel

Research Institute, Santa Creu i Sant Pau Hospital,

Barcelona, Spain

X. Palomer · D. Mauricio

Endocrinology and Nutrition Service,

Santa Creu i Sant Pau Hospital,

Barcelona, Spain

L. Calpe-Berdiel · F. Blanco-Vaca $(\bowtie)$

Biochemistry Service, Santa Creu i Sant Pau Hospital, Sant Antoni Maria Claret 167,

08025 Barcelona, Spain

e-mail: fblancova@hsp.santpau.es

Tel.: +34-93-2919451

Fax: +34-93-2919196

J. Verdaguer $\cdot$ J. Carrillo $\cdot$ X. Pastor

Immunology Laboratory for Research and Diagnosis,

Germans Trias i Pujol University Hospital,

Barcelona, Spain

D. Mauricio

Parc Taulí University Institute,

Sabadell, Spain inflammatory and immunomodulatory properties $[1,2]$. These properties have recently been studied in two different murine models of autoimmunity: one of experimental autoimmune encephalomyelitis [3] and one of collagen-induced arthritis [4]. Atorvastatin and simvastatin significantly suppressed autoimmunity in these respective animal models by inhibiting pathological pro-inflammatory responses. In both cases, evidence was found of a shift from a T helper type 1 (Th1) to a Th2 cell response [3, 4]. There is also evidence that atorvastatin can mediate a modest, but clinically apparent, anti-inflammatory effect in patients with rheumatoid arthritis [5].

The development of type 1 diabetes needs to be prevented. Previous studies have shown that $1 \alpha, 25$-dihydroxyvitamin $\mathrm{D}_{3}$ or nicotine treatments in mice arrested the progression of type 1 diabetes with a concomitant, potentially related inhibition of $\mathrm{Th} 1 \mathrm{CD} 4^{+} \mathrm{T}$ cell infiltration of the pancreas $[6,7]$. To gain preliminary insight into the potential usefulness of statins in patients with prediabetes, we sought to determine whether atorvastatin could delay, or even prevent, diabetes onset in two experimental models of type 1 diabetes: multiple low-dose streptozotocin and spontaneous non-obese diabetic (NOD) mice.

Female NOD mice were purchased from Taconic (Bomholt, Denmark) and maintained under pathogen-free conditions, and 4- to 5-week-old C57BL/6 male mice were purchased from Charles River Laboratories (Santa Perpètua de la Mogoda, Spain). The mice were fed an irradiated and non-irradiated regular chow diet (Harlan Ibérica, Sant Feliu de Codines, Spain), respectively, with or without $0.01 \%(\mathrm{w} / \mathrm{w})$ atorvastatin (Zarator; Pfizer, Madrid, Spain), equivalent to an approximate daily dose of $10 \mathrm{mg}$ of atorvastatin per $\mathrm{kg}$ of body weight. Diabetes was induced in the C57BL/ 6 mice by i.p. injection of $60 \mathrm{mg} / \mathrm{kg}$ streptozotocin in citrate buffer, $\mathrm{pH} 4.5$, for 5 consecutive days. In a separate experiment, atorvastatin was suspended in phosphate buffer and administered once daily by i.p. injection to streptozotocin-treated mice at a dose of $20 \mathrm{mg} / \mathrm{kg}$. Nonfasting blood glucose was monitored weekly and diabetes was diagnosed when glucose levels exceeded $11.1 \mathrm{mmol} / \mathrm{l}$ in two consecutive tests. 
Atorvastatin did not significantly reduce the incidence of diabetes or delay its onset, despite the different models, doses and administration routes tested. Neither the oral nor the i.p. atorvastatin treatment reduced the incidence or severity of diabetes in streptozotocin-induced mice, as indicated by the kinetics of hyperglycaemia development (Fig. 1a, b). Atorvastatin also failed to prevent diabetes
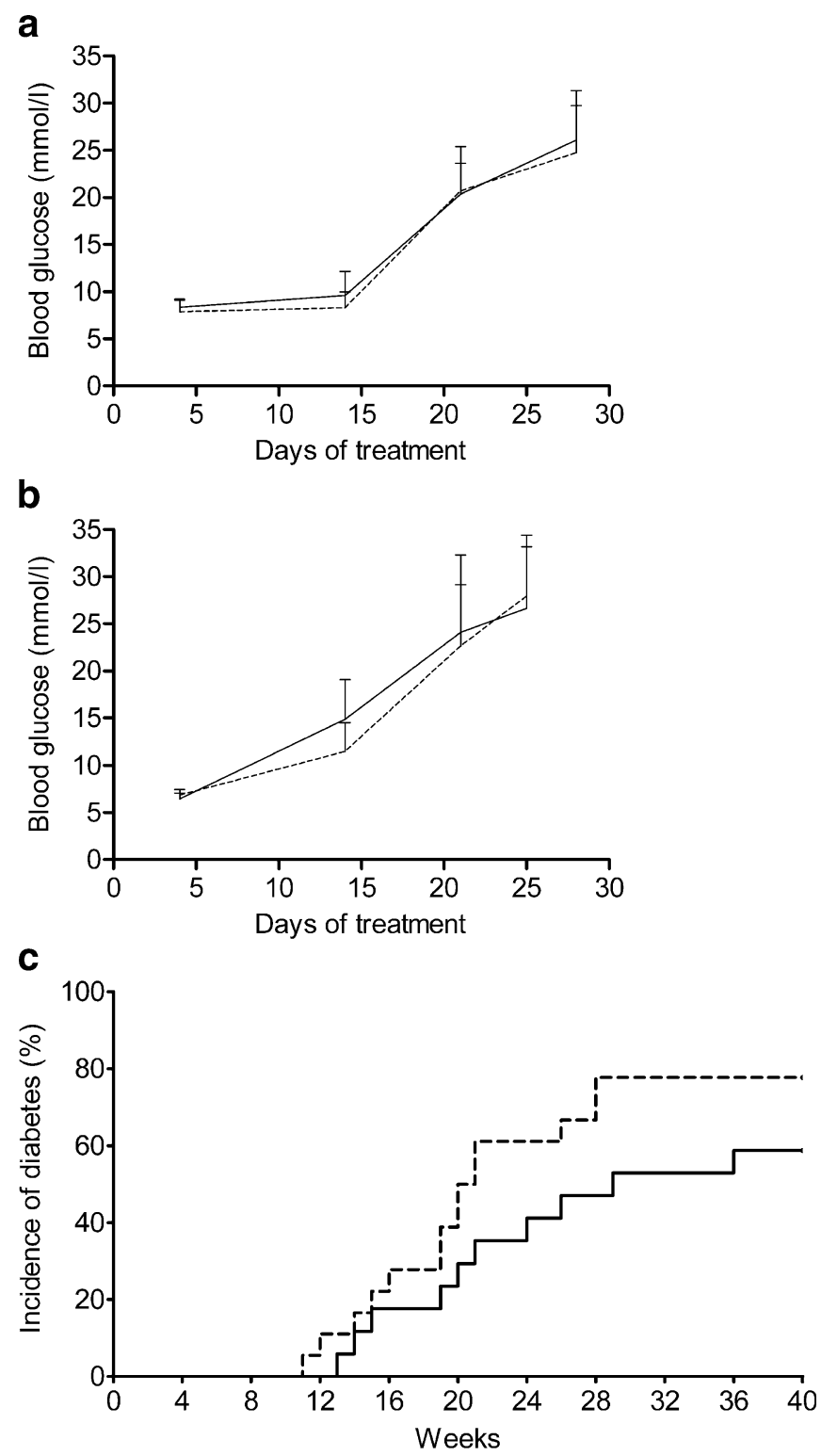

Fig. 1 Effect of atorvastatin on the development of autoimmune diabetes in mice. Blood glucose levels in streptozotocin-treated C57BL/6 male mice after treatment from day 0 (a) with or without $0.01 \%$ dietary atorvastatin ( $n=8$ per group), or (b) with $20 \mathrm{mg} / \mathrm{kg}$ atorvastatin in phosphate buffer, $\mathrm{pH} 7.4$, or phosphate buffer vehicle alone injected i.p. ( $n=6$ per group). c Cumulative incidence of diabetes in NOD female mice fed with or without $0.01 \%$ atorvastatin $(n=18$ per group). Data are presented as means \pm SD. The MannWhitney $U$ unpaired $t$-test was used to compare the experimental groups. A $p$ value of less than 0.05 was considered significant. Solid lines, vehicle-treated mice; dashed lines, atorvastatin-treated mice development in NOD mice (Fig. 1c). In the NOD mice, the first case of overt diabetes was seen at week 12 in the atorvastatin-treated group and at week 14 in the control group. The disease rate increased continuously thereafter up to 78 and $59 \%(p=0.17)$, respectively, by week 40 .

Our data are consistent with those obtained by Kanda et al. [8] who reported a slight, but significantly worse, glucose tolerance in streptozotocin-induced diabetic rats treated with atorvastatin or pravastatin.

The lack of effect of atorvastatin in the present study could be explained by the low dose used. Some authors argue that achievement of pleiotropic effects in mice may require a dose of up to $40 \mathrm{mg} \cdot \mathrm{kg}^{-1} \cdot \mathrm{day}^{-1}$ [4]. These doses are much higher than those used in man, but could be necessary in murine studies as a result of the rapid upregulation of $\mathrm{HMG}-\mathrm{CoA}$ reductase in response to the treatment [4]. However, it has been reported that the minimum dose required to induce an immunomodulatory effect in murine experimental autoimmune encephalomyelitis is $0.1 \mathrm{mg} / \mathrm{kg}$, which is clearly below the dose administered in our mice [3].

An effect of food intake cannot be ruled out either, since food ingestion decreases the bioavailability of fluvastatin, pravastatin and atorvastatin in humans [9], although plasma atorvastatin concentrations were not measured in our treated mice. However, no differences existed in food intake between the groups of animals being compared; furthermore, i.p. injections of atorvastatin were also unable to prevent diabetes in streptozotocin-treated mice.

The administration period for atorvastatin was comparable to that for other immunomodulatory treatments, such as $1 \alpha, 25$ dihydroxyvitamin $\mathrm{D}_{3}$ and nicotine, which have previously been reported to ameliorate or reduce diabetes incidence in mice $[6,7]$, thus ruling out the possibility of an insufficient period of treatment.

Other types of effects of statins on diabetes are also possible. Pravastatin reduced the risk of the development of type 2 diabetes by $30 \%$ in the West of Scotland Coronary Prevention Study (WOSCOPS) [4]. This effect could have been caused by a statin-induced decrease in plasma triglyceride, oxidative stress and inflammation in these patients $[10,11]$. However, we believe that, at this point in time, the potential effect of statins on type 2 diabetes prevention could be better addressed by clinical studies than by animal experimentation.

In conclusion, we were unable to substantiate any protective effect of atorvastatin, administered orally or i.p., on diabetes development in mouse models of type 1 diabetes.

Acknowledgements This study was supported in part by grants FIS C03-08, FIS G03/212 and Pfizer, Spain.

Duality of Interest

The authors declare that there is no duality of interest. 


\section{References}

1. McFarlane SI, Muniyappa R, Francisco R, Sowers JR (2002) Pleiotropic effects of statins: lipid reduction and beyond. J Clin Endocrinol Metab 87:1451-1458

2. Blanco-Colio LM, Tuñón J, Martín-Ventura JL, Egido J (2003) Anti-inflammatory and immunomodulatory effects of statins. Kidney Int 63:12-23

3. Youssef S, Stüve O, Patarroyo JC et al (2002) The HMG-CoA reductase inhibitor, atorvastatin, promotes a Th2 bias and reverses paralysis in central nervous system autoimmune disease. Nature 420:78-84

4. Leung BP, Sattar N, Crilly A et al (2003) A novel antiinflammatory role for simvastatin in inflammatory arthritis. J Immunol 170:1524-1530

5. McCarey DW, McInnes IB, Madhok R et al (2004) Trial of atorvastatin in rheumatoid arthritis (TARA): double-blind, randomised placebo-controlled trial. Lancet 363:2015-2021

6. Gregori S, Giarratana N, Smiroldo S, Uskokovic M, Aldorini L (2002) A 1alpha,25-dihydroxyvitamin $\mathrm{D}(3)$ analog enhances regulatory T-cells and arrests autoimmune diabetes in NOD mice. Diabetes 51:1367-1374
7. Mabley JG, Pacher P, Southan GJ, Salzman AL, Szabo C (2002) Nicotine reduces the incidence of type I diabetes in mice. J Pharmacol Exp Ther 300:876-881

8. Kanda M, Satoh K, Ichihara K (2003) Effects of atorvastatin and pravastatin on glucose tolerance in diabetic rats mildly induced by streptozotocin. Biol Pharm Bull 26:1681-1684

9. Veillard NR, Mach F (2002) Statins: the new aspirin? Cell Mol Life Sci 59:1771-1786

10. Paolisso G, Barbagallo M, Petrella G et al (2000) Effects of simvastatin and atorvastatin administration on insulin resistance and respiratory quotient in aged dyslipidemic non-insulindependent diabetic patients. Atherosclerosis 150:121-127

11. Tan KC, Chow WS, Tam SC, Ai VH, Kam CH, Lam KS (2002) Atorvastatin lowers C-reactive protein and improves endothelium-dependent vasodilatation in type 2 diabetes mellitus. J Clin Endocrinol Metabol 87:563-568 